\title{
Licensed Acupuncturists Join the Veterans Health Administration
}

\author{
Juli L. Olson, DC, MAOM
}

\begin{abstract}
This article introduces changes occurring in the Veterans Health Administration (VHA) with respect to the delivery of acupuncture. The VHA has published a new occupation code and job qualification standard allowing licensed acupuncturists to practice at the VHA medical centers. This policy shift comes at a time of great need for complementary and integrative health $(\mathrm{CIH})$ options for veterans as identified by the advancing research, policies, and legislation that support $\mathrm{CIH}$. The VHA initiatives include fostering an understanding of the distinct profession of licensed acupuncturists, developing an appreciation of the emerging evidence for acupuncture, and creating the cultural shift to support a wider view of CIH services. Historically, acupuncture was provided in the VHA mostly by physician-acupuncturists and chiropractic acupuncturists. The publication of a qualification standard for licensed acupuncturists allows the VHA to increase its provider base and create cost savings for the delivery of acupuncture. This move requires overcoming barriers to the integration of licensed acupuncturists into the VHA system. The goal is to increase the utilization of acupuncture among veterans.
\end{abstract}

Keywords: acupuncture, policy, U.S. Department of Veterans Affairs

\section{INTRODUCTION}

\section{A} LTHOUGH ACUPUNCTURE HAS BEEN PRACTICED to SOMP degree in the Veterans Health Administration (VHA) for years, February 7, 2018, marked a turning point in the delivery of acupuncture services. On that date, the VHA began recognizing licensed acupuncturists as having a standalone profession.

The publication of the VA Handbook $5005 / 100^{1}$ introduced the Acupuncture Qualification Standard, allowing facilities to hire licensed acupuncturists to provide acupuncture services. This came after almost 10 years of work by a dedicated group of interested healthcare professionals, creating a job classification and qualification standard for licensed acupuncturists.
In addition to offering this choice to veterans for the type of care they receive, another goal of this increased access to acupuncture is to effect cost savings throughout the VHA, due to the need for internal and external efficiency in an environment of increased demands and expenses. Moving to this point in recognizing licensed acupuncturists, however, was not without its barriers.

A small number of licensed acupuncturists had practiced in the VHA for years, working as contracted healthcare professionals not hired directly by the VHA, or as under a second license type held by the provider (RN, PA, DC) with a scope of practice that included acupuncture. Acupuncturists have also been known to volunteer their time in facilities to provide care to veterans. In 2015, 11 sites offered acupuncture by providers who were working without compensation. ${ }^{2}$

\footnotetext{
Veterans Health Administration, Des Moines, IA.
}

The views expressed are the private views of the author and do not reflect the official policy or position of the U.S. Department of Veterans Affairs, the Veterans Health Administration, the Department of Defense, or the United States Military. 
To date, physician-acupuncturists and chiropractic acupuncturists have provided most of the acupuncture offered in VHA medical centers. The shift to utilizing licensed acupuncturists will provide cost savings in salary differentials and allow greater access to acupuncture services. Most facilities that utilize physician-acupuncturists and chiropractic acupuncturists do not have full-time acupuncture services from these providers, as they typically split their time between practicing acupuncture and their other disciplines. In fiscal year 2017, more than 185,000 acupuncture treatments were provided to patients at VHA medical centers.

Currently, demand for acupuncture outpaces availability within many facilities. According to the VHA's Healthcare Analytics \& Information Survey (HAIG), acupuncture was one of the top three requested complementary and integrative health $(\mathrm{CIH})$ services by veterans (these services were acupuncture, chiropractic, and massage therapy). ${ }^{2}$ To meet the demand for care, the VHA utilizes care in the community to offer acupuncture through programs such as the Veterans Choice Program. In 2017, more than 47,000 veterans were authorized to receive acupuncture care outside VHA medical centers. By bringing acupuncture services back into the VHA medical centers, a significant cost savings could be realized.

The American Academy of Medical Acupuncturists recognizes 477 board-certified physician-acupuncturists. ${ }^{3}$ Given that many practicing physician-acupuncturists are trained but not board-certified, this small number of practitioners exemplifies the need to broaden the availability of practitioners. As of 2017, the National Certification Commission for Acupuncture and Oriental Medicine (NCCAOM) had almost 17,000 certified acupuncturists. ${ }^{4}$ As some states do not require NCCAOM certification, the number of licensed acupuncturists in the United States is also expected to be higher.

\section{DISCUSSION}

\section{The Need for Acupuncture Growth}

Veterans' healthcare needs are particularly suited to acupuncture care. A 2017 survey conducted by the National Institutes of Health concluded that $65.6 \%$ of veterans reported pain in the 3 months prior to the survey. ${ }^{5}$ Emerging needs for nonpharmacologic pain care have made expanding acupuncture services to veterans vital. VHA initiatives and legislative actions have furthered the need to increase this $\mathrm{CIH}$ service.

The VHA's Opioid Safety Initiative (OSI) highlighted concerns about the use of opioids by veterans to manage pain. ${ }^{6}$ The use of long-term opioid therapy to control pain increased $74 \%$ from 2004 to 2012 . The study revealed that $33 \%$ of veterans were opioid users, a percentage that was much higher than opioid use in the general public. The OSI recommends a stepped care model with nonpharmacologic pain care to include psychosocial interventions, CIH services, rehabilitation, and exercise.
In response to the 2016 Comprehensive Addiction and Recovery Act, $^{7}$ the VHA is funding 18 design sites to implement a Whole Health System, including the provision of selected CIH services. These design sites are tasked with developing best practices in transforming healthcare within the VHA from a disease-based model of healthcare to a patient-centered system.

The VHA also published Directive 1137: Provision of Complementary and Integrative Health $(\mathrm{CIH}),{ }^{8}$ calling for eight CIH modalities-including acupuncture-to be covered in the Veteran's Medical Benefits Package. This inclusion of acupuncture in the directive will require a greater resource allocation to acupuncture services.

To meet the demand for acupuncture more easily, Battlefield Acupuncture (BFA) continues to be supported throughout the VHA as an acupuncture therapy. Along with the Department of Defense (DoD), the VHA has been a leader in privileging various occupation types to perform this auricular acupuncture protocol. This technique-devised by Air Force Col. (ret) Richard C. Niemtzow, MD, $\mathrm{PhD}, \mathrm{MPH}$ - was promoted widely in the VHA and DoD through a Joint Incentive Fund (JIF). Under the JIF, 88 medical providers were trained in medical acupuncture and BFA training was conducted throughout the DoD and VHA. Today, the VHA has more than 1800 providers who are trained to offer BFA to veterans.

\section{The Acupuncture Qualification Standard}

Developing the acupuncture qualification standard greatly increases the ability of the VHA to deliver cost-effective acupuncture services. As defined in the VHA Acupuncture Qualification Standard, licensed acupuncturists hired by the VHA must have a masters or doctoral level degree in acupuncture or Oriental Medicine from a school accredited by the Accreditation Commission on Acupuncture and Oriental Medicine (ACAOM), have current certification by the NCCAOM, and maintain licensure in a U.S. state or territory.

Education requirements defined by the ACAOM include a minimum of 3 academic years of study with 105 semester credits or 1905 hours of training for a masters in acupuncture (MAc) degree, or 146 semester credits or 2625 hours of training for a masters in acupuncture and Oriental Medicine (MAOM) degree. Biomedical clinical science makes up 30 semester units in the MAc program standard and 34 units in the MAOM standard.

The NCCAOM offers two levels of diplomate status for acupuncturists: diplomate in acupuncture or diplomate in Oriental Medicine. The diplomate in acupuncture requires board examinations in biomedicine, acupuncture with point location, and foundations of Oriental Medicine. The diplomate in Oriental Medicine includes a fourth board examination in Chinese herbology. The minimum qualification for the VHA is the diplomate in acupuncture. 
Prior to sitting for the NCCAOM board examinations, a candidate must be certified in clean needle technique for acupuncturists (CNT). This 8-hour certification class includes didactic and practical learning, and a written and practical examination, to determine the competency of an acupuncturist in following infection-control procedures. The course was originally designed in 1995 and has been updated continuously to follow the most current understanding of safe acupuncture practice. ${ }^{*}$

A current unrestricted license in a U.S. state or territory that licenses acupuncturists is also required for VHA employment. There are three states that currently do not license acupuncturists: Alabama; Oklahoma; and South Dakota. According to statistics available from the NCCAOM, only 62 diplomates are currently located in these states.

\section{Overcoming Barriers}

The inclusion of licensed acupuncturists in the VHA comes at a time when there is an increased need and interest in nonpharmacologic pain treatment. Some change in culture was required to shift a conventional medical system to being open to integrative health.

This began with educating health professionals about acupuncture's effectiveness data and safety profile. The Acupuncture Evidence Map was published in 2014. ${ }^{9}$ This map was created by an evidence-based synthesis project conducted by the Health Research \& Development arm of the VHA. This report was key in demonstrating how acupuncture could be of use to veterans with pain and mental health conditions.

The greater inclusion of acupuncture in the larger U.S. healthcare system was important for the culture shift happening within the VHA. For example, The Joint Commission clarified its pain-management recommendations for nonpharmacologic pain care for patients to include acupuncture in $2015 .^{10}$ The Joint Commission also emphasized this recommendation in 2018, making the availability of nonpharmacologic pain-care methods a scored element of performance for accredited hospitals. ${ }^{11}$

Multiple clinical guidelines now recommend acupuncture as a valid treatment option, including the:

- Department of Veterans Affairs/DoD clinical practice guidelines" (2017): Acupuncture may be offered for chronic low-back pain. ${ }^{6}$

- American College of Physicians clinical practice guidelines for acute, subacute, and chronic low-back pain (2017): Acupuncture may be used as a first-line treatment for nonradicular low-back pain. ${ }^{12}$

- American Society of Clinical Oncology clinical practice guidelines for chronic pain management in adult cancer survivors (2016): Clinicians may prescribe acupuncture for pain. ${ }^{13}$

\footnotetext{
*Visit ccaom.org to obtain a download of the CNT manual.
}

- Agency for Healthcare Research and Quality (2016). Acupuncture is effective for chronic low-back pain. ${ }^{14}$

Another barrier against the recognition of licensed acupuncturists in the VHA included difficulty with federal tracking and classification of the profession. When investigating the professional characteristic of the acupuncture profession, the Federal Bureau of Labor Statistics (BLS) information was incomplete, as acupuncturists were not tracked as having a separate and distinct profession.

It was discovered that the NCCAOM had been working since 2006 to create a BLS job classification for acupuncturists. Data collected in the NCCAOM job analysis was instrumental in meeting the BLS requirements that a new profession was separate and distinct and growing. In July 2016, the BLS agreed to add acupuncture to the 2018 edition of the BLS Handbook. ${ }^{15}$

\section{CONCLUSIONS}

At a time when acupuncture evidence is on the rise, and the need for complementary and integrative practices is highlighted, the VHA has taken a significant step to provide greater access to acupuncture services for veterans. This change provides an opportunity to advance acceptance of acupuncture and to examine its utilization and effectiveness in the largest healthcare system in the United States.

Acupuncture program support at the VHA is housed in the Office of Patient Centered Care and Cultural Transformation under the direction of the Integrative Health Coordinating Center. These offices are charged with changing the culture of care at the VHA from that of "sick care" to whole healthcare.

\section{AUTHOR DISCLOSURE STATEMENT}

No competing financial interests exist.

\section{REFERENCES}

1. Department of Veterans Affairs. Part II: Appendix G53. Acupuncturist qualification standard GS-601. Veterans Health Administration. In: VA_Handbook_5005_100_Certified.pdf Washington DC: Department of Veterans Affairs; February 7, 2018;II-G53-1-II-G53-8.

2. Office of the ADUSH for Policy and Planning, Healthcare Analysis \& Information Group (HAIG). FY 2015 VHA Complementary and Integrative Health $(\mathrm{CIH})$ Services (formerly CAM). 2015. Online document at: https://sciencebasedmedicine .org/wp-c3ntent/uploads/2016/07/FY2015_VHA_CIH_signed Report.pdf Accessed April 20, 2018.

3. American Academy of Medical Acupuncturists (AAMA). About AAMA. Online document at: www.medicalacupuncture .org Accessed April 20, 2018. 
4. National Certification Commission for Acupuncture and Oriental Medicine (NCCAOM). National Certification Commission for Acupuncture and Oriental Medicine. Online document at: http://www.nccaom.org/visitors/state-regulators/ Accessed April 20, 2018.

5. Nahin RL. Severe pain in veterans: The impact of age and sex, and comparisons to the general population. J Pain. 2017; 18(3):247-254.

6. U.S. Department of Veterans Affairs. Management of Opioid Therapy (OT) for Chronic Pain (2017)_VA/DoD Clinical Practice Guidelines. Online document at: www.healthquality .va.gov/guidelines/pain/cot/ Accessed April 20, 2018.

7. The Public Health and Welfare Comprehensive Addiction and Recovery Act of 2018, 42 U.S.C. 290bb-25g. Online document at: http://uscode.house.gov/view.xhtml?req=(title:42\%20 section:290bb-25g\%20edition:prelim) Accessed April 20, 2018.

8. Veterans Health Administration. VHA Directive 1137: Provision of Complementary and Integrative Health (CIH). 2017. Online document at: www.va.gov/VHAPUBLICAtIONs/View Publication.asp?pub_ID=5401 Accessed April 20, 2018.

9. Hempel S, Taylor SL, Solloway MR, et al. Evidence Map of Acupuncture: VAESP Project \#05-226. Washington, DC: Department of Veterans Affairs; 2014.

10. The Joint Commission. Clarification to Standard PC.01.02.07. 2015. Online document at: www.jointcommission.org/assets/ 1/18/Clarification_of_the_Pain_Management__Standard.pdf Accessed April 20, 2018.

11. The Joint Commission. New and Revised Standards Related to Pain Assessment and Management: LD.04.03.13. 2018. Online document at: www.jointcommission.org/assets/1/18/
R3_15_Pain_Assess_Mgmt_CAH_6_22_18_FINAL.pdf Accessed April 20, 2018.

12. Qaseem A, Wilt TJ, McLean RM, Forciea MA; Clinical Guidelines Committee of the American College of Physicians. Noninvasive Treatments for Acute, Subacute, and Chronic Low Back Pain: A Clinical Practice Guideline from the American College of Physicians Clinical Guidelines Committee. Ann Intern Med. 2017;166(7):514-530.

13. Paice JA, Portenoy R, Lacchetti C, et al. Management of Chronic Pain in Survivors of Adult Cancers: American Society of Clinical Oncology Clinical Practice Guideline. J Clin Oncol. 2016;34(27):3325-3345.

14. Chou R, Deyo R, Friedly J, et. al. Comparative effectiveness reviews. In: Agency for Healthcare Research and Quality (AHRQ). Noninvasive Treatments for Low Back Pain [Internet]. Rockville, MD: AHRQ; 2016:169.

15. National Certification Commission for Acupuncture and Oriental Medicine. New Independent SOC for Acupuncturists Proposed by the BLS for 2018. Bureau of Labor Statistics. Online document at: http://www.nccaom.org/resource-center/ bls-timeline/ Accessed April 20, 2018.

Address correspondence to: Juli L. Olson, DC, MAOM Veteran's Health Administration 3600 30th Street Des Moines, IA 50310 E-mail: juli.olson@va.gov 